\title{
Article \\ Evaluation of Development Level and Technical Contribution of Recent Technologies Adopted to Meet the Challenges of 5G Wireless Cellular Networks
}

\author{
Saleh Albadran
}

Citation: Albadran, S. Evaluation of Development Level and Technical Contribution of Recent Technologies Adopted to Meet the Challenges of $5 \mathrm{G}$ Wireless Cellular Networks. Symmetry 2021, 13, 635. https:// doi.org/10.3390/sym13040635

Academic Editors: Sun Young Cho and Sergei D. Odintsov

Received: 16 February 2021

Accepted: 7 April 2021

Published: 9 April 2021

Publisher's Note: MDPI stays neutral with regard to jurisdictional claims in published maps and institutional affiliations.

Copyright: (C) 2021 by the author. Licensee MDPI, Basel, Switzerland. This article is an open access article distributed under the terms and conditions of the Creative Commons Attribution (CC BY) license (https:/ / creativecommons.org/licenses/by/ $4.0 /)$.
Department of Electrical Engineering, College of Engineering, University of Hail, Hail 55211, Saudi Arabia; s.abadran@uoh.edu.sa

\begin{abstract}
The evolution of the global wireless market is accompanied by an increased need in terms of speed and number of users, lower latency, better coverage, better spectral efficiency and quality of service, etc. To meet these needs, $5 \mathrm{G}$ has recently been introduced as an effective solution which targets, via the large scale deployment of symmetric antennas, a wide variety of sectors such as energy, health, media, industry, transport and especially wireless cellular networks which are among the most important pillars of modern societies. Multiple Input, Multiple Output (MIMO) systems, which have been extended to "Massive MIMO" mode and which consist of increasing the number of radiating elements involved in the transmission and reception of the radio link, are a very promising solution for improving the spectral efficiency of wireless communication systems (WCSs). Motivated by the aforementioned developments, the present paper investigates the increased capacity of MIMO systems to improve transmission in WCSs using 5G. It carefully focuses on the evaluation of the development level and technical contribution of MIMO systems and millimeter wave (mmWave) bands in $5 \mathrm{G}$ wireless cellular networks (WCNs) and gives important recommendations.
\end{abstract}

Keywords: wireless cellular networks; symmetric networks; MIMO systems; 5G; multi-user MIMO systems; Massive MIMO; mmWave

\section{Introduction}

Since the 1980s, when the first analog communication systems appeared, a succession of new and more advanced systems were developed and introduced each decade to improve quality of service (QoS). In recent years, the telecommunications sector has experienced spectacular developments marked by the emergence of different technologies, making it possible to communicate in increasingly more efficient ways. From the initial transport of voice between two users, continuous efforts in this area have succeeded in sharing data which can take many forms: messages, texts, photos, videos, etc. Most significantly, the use of mobile networks has become more and more spread worldwide.

According to the Cisco company, global mobile data traffic has exceeded in 2017 its 2012 level by a factor of 12.6. This phenomenal growth continues today and the total mobile traffic has increased by a factor of ten between 2019 and 2022 [1]. Therefore, the future cellular infrastructure has to be ready to support new usages and specially to meet modern wireless communication standards and users' satisfaction. In particular, the last decade has seen a dramatic increase in mobile data traffic due to the wide deployment of the use of machine-to-machine (M2M) communications systems and smart devices in most applications/sectors. According to recent forecasts by the International Telecommunication Union (ITU), the increase in the volume of global mobile traffic between 2010 and 2030 will be by more than 660 times (Figure 1) [1]. 


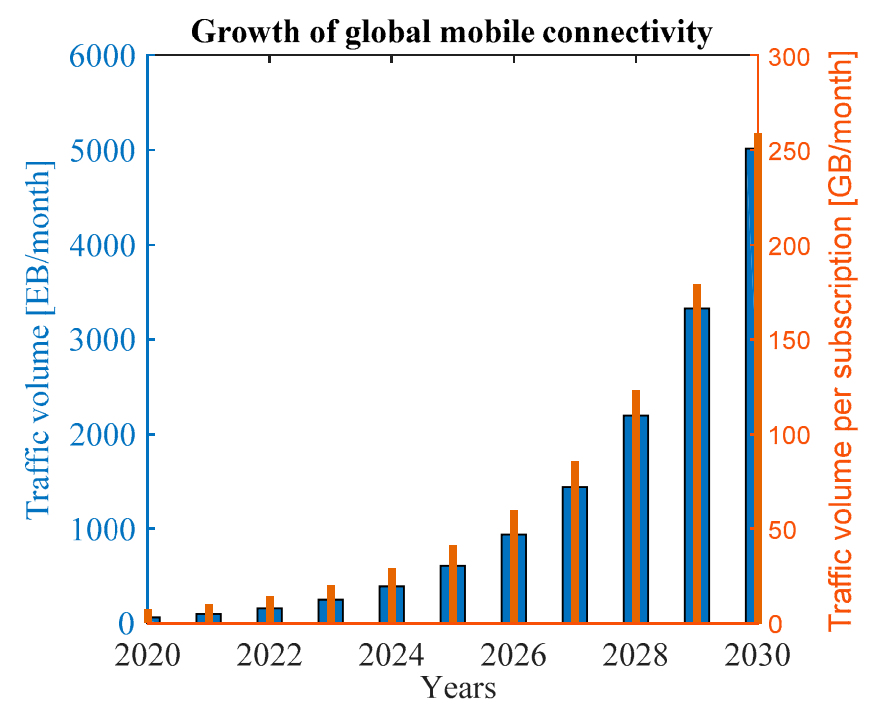

Figure 1. Growth of global mobile connectivity.

In fact, various standards for cellular networks, from $1 \mathrm{G}$ to $5 \mathrm{G}$ via $2 \mathrm{G}, 3 \mathrm{G}$ and $4 \mathrm{G}$, have been introduced [2]. According to Figure 2, which shows the various wireless communication network technologies and their main characteristics, 2G, 3G and 4G generations have become unable to respond optimally to the constraints of speed, technology, frequency, data capacity, latency, etc. [3]. This is due to the frequency selectivity of the channels and the multiple paths that may be taken by the same signal $[4,5]$.

Faced with these challenges, 5G wireless communications have the capability to satisfy the objectives concerning the transmission of digital information at higher speeds, increased demands on the quality of service and the resolution of the problem of limited network capacity compared to the bandwidth or the transmission power [6]. To achieve these objectives, 5G combines several techniques, amongst them the multi-antenna technique (Multiple Input, Multiple Output (MIMO)) which allows increasing the capacity of communication systems based on the installation of several antennas, having a symmetrical structure, for transmission and reception. This allows the system to be more robust against the fading of the transmission channel since it reduces the energy consumption and exploits the spatial diversity in a better way, and thus improves the quality of transmission $[7,8]$.

In this regard, it would be very important to conduct a detailed investigation of all the techniques used to further enhance the performance of 5G wireless cellular networks and propose methodologies to assess their efficiency. Particular attention will be paid to the evaluation of the contribution of MIMO systems and other new technologies to satisfy the various needs facing $5 \mathrm{G}$ wireless communications and determine the best solutions that serve as reduced-complexity guides on future research directions for $5 \mathrm{G}$ and future wireless communications. 


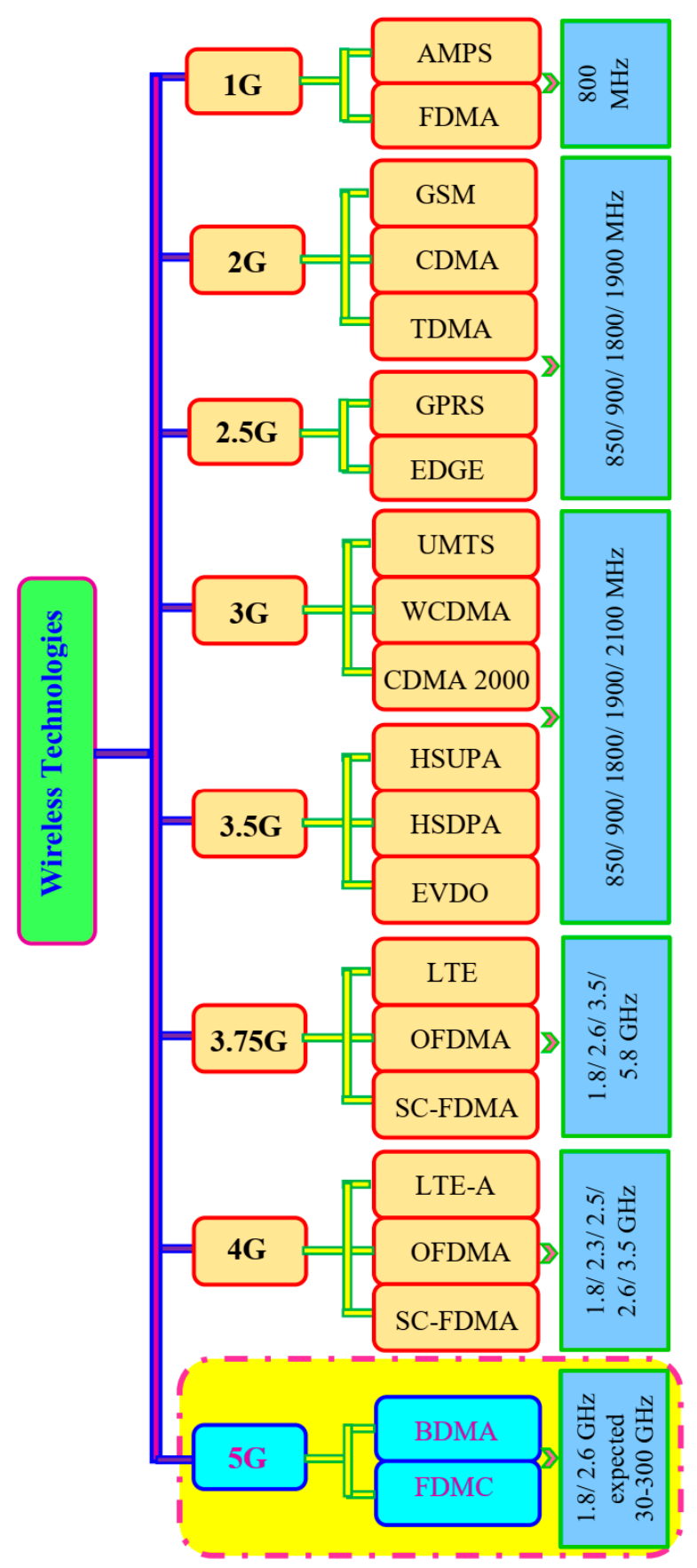

Figure 2. Wireless communication network technologies: Access technologies and frequency bands.

\section{Related Works}

From one year to the next, the demand for speed and quality of service for wireless communications continues to grow. The need for instantaneity and interaction in exchanges, the integration of multimedia services of different forms with variable bit rates and of high quality requires performance that can meet user expectations [9]. On the other hand, the number of wireless terminals is constantly increasing, which implies the coexistence of users in the same environment. The multitude of communication standards and protocols make the radio spectrum a highly congested resource where the number of interferers increases considerably [10]. Current techniques associated with suitable modulations and coding implementing a point-to-point link have approached the theoretical limit of Shannon [11]. However, the spectral efficiency acquired by these techniques has not yet managed to overcome the limits of the performances that they must satisfy. Recently, 
considerable improvements in the theoretical capacity of broadcaster-rich channels, such as wireless local area networks (WLAN1), have been achieved with multi-antenna MIMO architectures for transmission and reception [12]. With the introduction of a new dimension, that of space, and the emergence of the field of space-time processing, the contributions are essentially the achievement of a theoretical capacity exceeding the Shannon limit and the improvement of the quality of service by reducing signal fading by exploiting multiple paths [13].

With the increasing development in wireless communication networks (WCNs) and the emergence of the fifth generation (5G) mobile networks, MIMO systems have attracted widespread public attention for guaranteeing the resilient, reliable and cost-effective exploitation of 5G WCNs [14]. Nevertheless, and in order to alleviate the complexity of studying such systems, researchers have resorted in several cases to neglect several factors that can impact the practical execution and the accuracy of MIMO results. To overcome this, particular attention has been paid to MIMO systems; they are among the most popular topics in wireless communication and have reached a much focused spot on the modern "hottest wireless technology" list $[15,16]$. Various studies have taken an explicit interest in the question of estimating the direction of departures (DODs) and direction of arrivals (DOAs) for the source separation in MIMO systems [17]. Two main other subjects were dealt with: Multi-user MIMO systems (MU-MIMO) [18-20] and Massive MIMO systems [21]. In [22], the authors proposed a parametric method of antenna processing. This method, previously used in radar systems, is based on tensors algebra in the context of radio communications. According to comparative studies, this method is considered efficient enough for users with sufficient spacing and with a number of sources greater than the number of sensors. Furthermore, 5G is also appealed to be able to estimate a large number of channels and connect many users to the network at the same time by accessing the network uplink and identifying active users while minimizing latency with reduced spectrum and power overheads. To respond to these challenges, MU-MIMO systems have been introduced. Nejah et al. [19] proposed two types of authentication, one so-called blind and the other semi-blind, to identify active users and their channels, respectively. The proposed approach exploited the embedded signaling and, more precisely, the modulation constellations with a randomized rotated code for the uplink of MU-MIMO systems [19]. In addition, the study of a channel identification technique for a MU-MIMO system was carried out to estimate the directions of arrival/departure and to deduce therefrom the parameters of the impulse responses of the channel. In a different context, that of cognitive radio, the multidimensional model for estimating direction parameters was also deployed using a decision algorithm for channel allocation [23]. The effect of power amplifier (PA) nonlinearity on MU-MIMO orthogonal frequency division multiplexing (MU-MIMO-OFDM) systems has been also studied for a base station (BS) using linear precoding for data transmission in the case of single-antenna users through a correlated channel [24].

Massive MIMO systems have also been introduced as a key element of the present and future of 5G WCNs especially for a symmetric network characterized by a common number of antennas between all the transmitters and the same number of antennas for all the receivers. In this regard, the feasibility of a time-frequency estimation of the directions of arrival within the framework of a Massive MIMO system has been studied [9,21]. In addition, the pilot contamination problem was tackled to resolve this source separation problem in the time, frequency and space domains and allow the Massive MIMO base station to discriminate between its users and those of other cells and, subsequently, cancel the effect of pilot contamination. In [21], it has been proved that maximal energy efficiency can be achieved by a Massive MIMO setup wherein hundreds of antennas are installed to serve a quite large number of users who use zero-forcing (ZF) processing.

Despite these advantages, the development of Massive MIMO systems faces many drawbacks. Indeed, the search for solid support for various Internet of things (IoT) services is the basis of the development of $5 \mathrm{G}$ mobile networks. This is because IoT services rely 
heavily on the new radio (NR) for which many air interfaces are designed for the future generation Node B (gNB) and user equipment (UE). Consequently, a new technique called Massive MU-MIMO is emerging as a favorable solution to satisfy the tremendous demand for high-speed wireless networks. In the literature, this technique is attracting the attention of many researchers $[22,25,26]$. In [27], it has been shown that the emerging MU-MIMO concept is very efficient in IoT networks, even under such unfavorable conditions as random user's deployment and shadow fading because it succeeds in enabling ultrareliable and low-latency communications (URLLCs) with pilot-assisted channel estimation (PACE) and zero-forcing (ZF) detection. In addition, full duplex (FD) wireless transmission is considered at the heart of discussions on the definition of $5 \mathrm{G}$ given its ability to increase the user throughput and the spectral efficiency in WCNs [28].

In the same context of the exponential digitization of the world where everything has become connected, $4 \mathrm{G}$ based on the LTE (Long Term Evolution) standard and IP technology has encountered serious problems mainly linked to the overload of the frequency spectrum and of course to other deficits. To meet the current requirements and those of the future due to the growth of connected objects known by various categories of use, the new $5 \mathrm{G}$ standard has responded to the deficits related to the increased throughput or bandwidth and has achieved an ultra-short latency and reduced power consumption with ubiquitous quality service [29]. This has been accomplished by the introduction of different technologies and even by the combination of several of them [30,31].

In recent years, the maximum bandwidth allocated to all cellular technologies has been limited to $780 \mathrm{MHz}$ and, therefore, the release up of frequency bands has become a major priority to overcome the global bandwidth shortage. In this context, 5G WCNs use an extended frequency spectrum to cover medium and high frequencies integrating mmWave technology [32,33]. Within this framework, a simple, low-profile, compact, dualpolarization, low-cost and suspended patch antenna has been designed to operate in a 3.3-3.8 GHz band used for a 5G base station with a gain of $8.95 \pm 0.25 \mathrm{dBi}$ [34]. In [29,35], a Sub-6 GHz dual-band $8 \times 8 \mathrm{MIMO}$ antenna and Sub-6 GHz 5G NR broadband eightantenna array have been designed, respectively, for $5 \mathrm{G}$ smartphones. Unlike this antenna consisting of a monopole operating in 3.1-3.85 and 4.8-6 GHz for the low band and the high band respectively, another dipole frequency-reconfigurable antenna has shown two resonant modes centered at 3.5 and $5.5 \mathrm{GHz}$ or, to be more specific, two bandwidths that are 2.89-4.07 GHz and 5.1-6.19 GHz [36]. Otherwise, some researchers have dealt with cases treating the combination of mmWave and Sub-6 GHz for $5 \mathrm{G}$ antenna systems [30,37]. Here, it is important to mention that the use of mmWave technology is primarily motivated by the fact that the sub-bands $6 \mathrm{GHz}$ are almost saturated.

According to the conducted literature review, it has been widely proven that MIMO systems are contributing to satisfying the various needs facing $5 \mathrm{G}$ wireless communication systems. This paper is concerned with the evaluation of the development level and the technical contribution of various MIMO, mmWave and full duplex technologies in 5G wireless cellular networks.

To accomplish the goals and objectives, the rest of the paper will be as follows:

- To summarize the features of MIMO wireless technologies, beginning with the evolution of such systems from Single Input, Single Output (SISO) systems

- $\quad$ To discuss MIMO, MU-MIMO, Massive MIMO and Massive MU-MIMO techniques used to push $5 \mathrm{G}$ performance further and methodologies to assess their efficiency

- To evaluate the contribution of different MIMO systems, mmWave technology and full duplex, in terms of benefits, to satisfy the various needs facing $5 \mathrm{G}$ wireless communications as well as their challenges

- To provide a guide on future research directions, outline the substantial conclusions and suggest future works for $5 \mathrm{G}$ and beyond wireless cellular networks 


\section{MIMO Wireless Technologies Features}

\subsection{From SISO to MIMO}

Traditional communications systems have been based on only two antennas: one transmitting and the other receiving. With the necessity to keep up with the exponentially growing mobile communications service needs for transmitting more data, increasing transmission capacity through the use of multiple antennas has become a tangible reality. SISO, SIMO (Single Input, Multiple Output), MISO (Multiple Input, Single Output) and MIMO techniques have been proposed to allow data transfers [38]. In what follows, an overview of these main multiplexing techniques used in mobile networks is given.

\subsubsection{SISO Systems}

SISO systems are the simplest links as they use single antennas at the BS base stations (transmitters) and UEs (receivers).

For a SISO system with the gain of channel $h$ and a signal-to-noise ratio at the receiving antenna $\gamma$, and if we assume that the channel state information (CSI) is unknown, the capacity is expressed by:

$$
C=\log _{2}\left(1+\gamma|h|^{2}\right)
$$

Given that $E\left(\left|h_{i}\right|^{2}\right)=1$.

, the theoretical capacity (bits/s/Hz) is expressed by:

$$
C_{t}=\log _{2}(1+\gamma)
$$

\subsubsection{SIMO Systems}

The SIMO channel is a conventional multi-antenna system providing a channel or a transmission antenna and several reception antennas for the mobile phone.

For a SIMO system with one transmitting antenna and $N$ receiving antenna, if $h_{i}$ is the complex gain between the transmitting antenna and the $i$ th receiving antenna, its capacity is expressed as follows:

$$
C=\log _{2}\left(1+\gamma \sum_{i=1}^{N}\left|h_{i}\right|^{2}\right)
$$

Its Shannon capacity is:

$$
C_{t}=E(C)=\log _{2}\left(1+\gamma N^{2}\right)
$$

\subsubsection{MISO Systems}

The MISO channel model is basically the reverse of SIMO. It uses several transmit antennas (BS) and a single receive antenna for the mobile phone.

\subsubsection{MIMO Systems}

Continued efforts to increase performance, in terms of improving capacity, increasing spectral efficiency and minimizing the effects of multipath interference, gave rise to the discovery of MIMO systems. These MIMO systems are more efficient than their predecessors from a capacity and reliability point of view thanks to the important advantages related mainly to multiplexing, diversity and array gains [27,39].

For a MIMO system including $M$ transmitting antenna and $N$ receiving antenna, the matrix $H$ is given by:

$$
H=\left[\begin{array}{ccccc}
h_{11} & h_{12} & \cdot & \cdot & h_{1 M} \\
h_{21} & h_{22} & \cdot & \cdot & \cdot \\
\cdot & \cdot & \cdot & h_{i j} & \cdot \\
\cdot & \cdot & \cdot & \cdot & \cdot \\
h_{N 1} & h_{N 2} & \cdot & \cdot & h_{N M}
\end{array}\right]
$$


Depending on the number of the transmitting antenna $i$ and that of the receiving antenna $j, h_{i j}$ is the complex gain of the channel between these antennas; hence, the capacity of the MIMO system is established by Equation (6):

$$
C=\log _{2}\left(\operatorname{det}\left[I_{N}+\frac{\gamma}{M} H H^{H}\right]\right)
$$

As depicted in Figure 3, simulations have been developed to compare the performance of these different systems in terms of capacity vs. signal to noise ratio (SNR) [2]. It has been found that the capacity of MIMO systems outperforms all other systems. Furthermore, this capacity increases with the increase in the SNR, of course, when the number of antennas on the transmitter and the receiver sides increases.

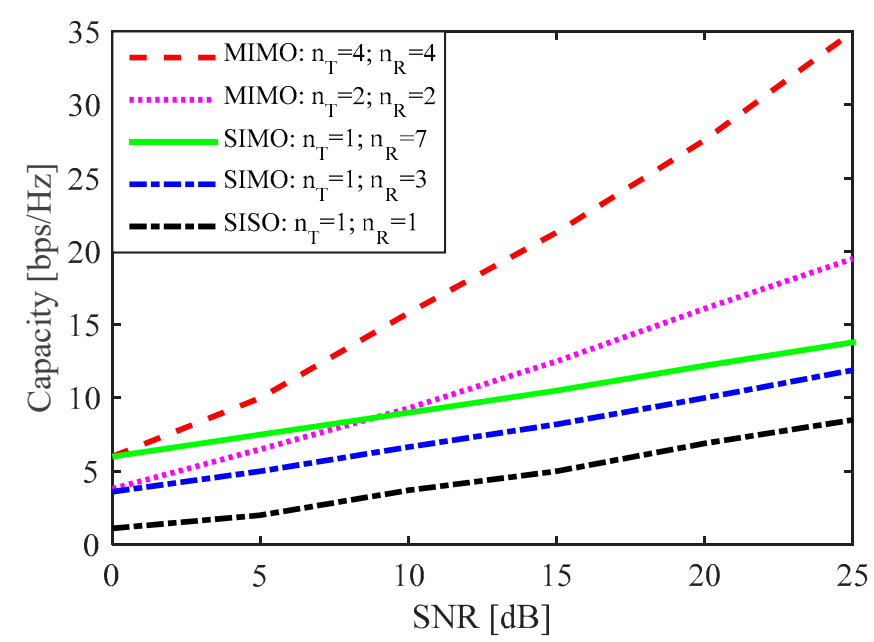

Figure 3. Capacity of Single Input, Single Output (SISO), Single Input, Multiple Output (SIMO) and Multiple Input, Multiple Output (MIMO) systems vs. signal to noise ratio (SNR) for different numbers of antennas.

\subsection{Recent Wireless Technologies: From MIMO to Massive MIMO}

From their inception, MIMO systems have brought various advantages that have proven their effectiveness, in terms of capacity, range, etc., to wireless communications networks. MIMO systems have mainly taken advantage of the multi-path environment, ensuring the increase in transmission capacity and obtaining independent signals on the antennas by using the different propagation channels created by diffraction and/or by reflection of waves.

Despite these successes, various new levels of complementary processing are always being improved upon, which aim to enable MIMO wireless communication systems to achieve more purposes. Regarding the techniques of encoding signals in a MIMO system, three main approaches can be distinguished:

\subsubsection{Techniques of Encoding Signals in a MIMO System}

- $\quad$ Multiplexing by orthogonal frequency division (OFDM):

OFDM is the basis of most broadband transmission applications. It consists of dividing the signal to be transmitted on numerous carriers, orthogonal to each other, and at different frequencies [40].

The use of OFDM with MIMO systems makes it possible to have high speed communications without interference between the transmitted signals and with the possibility of recovering information lost on other carrier frequencies. Nonetheless, OFDM requires frequency and time synchronization and the estimation of the MIMO channel of MIMOOFDM systems to constantly ensure orthogonality between carriers which can easily be damaged by OFDM's sensitivity to carrier frequency error. 
- $\quad$ Spatial Division Multiplexing (SDM):

It is an approach capable of increasing the data throughput and improving bandwidth utilization. Indeed, during SDM, several independent data streams are simultaneously multiplexed in a spectral channel [41]. To achieve this, an analog-to-digital converter and a different RF radio-frequency chain are necessarily used in a MIMO system for each antenna. In addition, each spatial stream must have its own pair of transmit/receive antennas at each end of the radio link. The MIMO-SDM combination is found to be cost effective as it has been characterized by inherent security at the physical layer [42].

- $\quad$ Space-Time Block Code (STBC):

The STBC is able to send different signals on each antenna, as in the case of SDM. The STBC manages to improve the robustness of the receiver through the diversity of the transmission channel and the introduction of information redundancy between the receiver and the transmitter. The STBC channel includes $\mathrm{M}^{*} \mathrm{~N}$ independent fading channels. On the one hand, the STBC technique is very efficient in the sense of overcoming the reduction in data transmission capacity because it does not impose any knowledge of the state of the channel (CSI) [43]. However, this method cannot, in any case, guarantee a linear relationship between the number of elements used and the transmission capacity. To achieve this, one of the solutions proposed for MIMO systems is to combine STBC with SDM. Due to the fact that they use several antennas, at the BS level as well as at the UE level, bringing advantages in relation to multiplexing, diversity and matrix gains, MIMO systems achieve better performance in terms of capacity and reliability compared to those of SISOs [44]. However, it is important to note that one of the concerns associated with MIMO operation is the existence of a trade-off between maximum diversity and multiplexing gains.

\subsubsection{MIMO Systems Techniques}

MIMO systems potentially increase the performance of wireless communications systems, in particular the range and reliability of links independent of the propagation channel. Consequently, this contribution of MIMO systems in terms of transmission rate and emission of independent information by appropriate spatial processing is very important because of its use in the majority of communication standards such as IEEE 802.11n and IEEE 802.16 [45,46] as well as Long term evolution (LTE) and its improved version LTE Advanced (LTEA) [47]. The use of these technologies began with the modest integration of MIMO links ( $\mathrm{Ne} \times 2)$ where $\mathrm{Ne} \geq 2$ in $4 \mathrm{G}$ mobile networks. However, it was with $5 \mathrm{G}$ that the integration of Massive MIMO systems with a high number, up to a few hundred, of antennas on the base station was achieved. In the literature [16,42,44], among MIMO technologies, it is important to draw attention to single-user MIMO (SU-MIMO), multi-user MIMO (MU-MIMO) configurations [48].

\section{- $\quad$ SU-MIMO}

The SU-MIMO system corresponds to a simple MIMO link between two points in space devoting all the time-frequency resources to one terminal, while exploiting the techniques of spatial multiplexing, emission diversity and beamforming $[49,50]$.

\section{- MU-MIMO}

The MU-MIMO system makes it possible to achieve gains compared to SU-MIMO especially in the event of spatial correlation between the channels. Indeed, this configuration corresponds to a set of MIMO links between one or more points in space on transmission and one or more points in space on reception using the same time-frequency resources [51,52]. Intuitively, MU-MIMO can increase the capacity or spectral efficiency of a transmission network without increasing the bandwidth [53]. 


\section{- $\quad$ Massive MIMO}

The transition between MU-MIMO and Massive MIMO occurs when the number of antennas on the base station ( $\mathrm{Ne}$ ) becomes very large (up to a few hundred) and exceeds that of users K [54-57]. Massive MIMO presents various advantages such as:

- $\quad$ increased spectral efficiency because many users are served at the same time

- $\quad$ increased energy efficiency because the energy may well be focused on a very small region of the space

- decreased latency because it is based on the law of large numbers to avoid fading.

The comparison of the various aforementioned MIMO configurations and their advantages in terms of multiplexing, diversity, and array gain are given in Table 1.

Table 1. Advantages of single-user MIMO (SU-MIMO), multi-user MIMO (MU-MIMO) and MassiveMIMO antenna technologies.

\begin{tabular}{|c|c|c|c|}
\hline & \multicolumn{3}{|c|}{ MIMO Antenna Technology } \\
\hline & SU-MIMO & MU-MIMO & Massive MIMO \\
\hline Multiplexing gain & (1) (1) & $(1)(1)$ & (1) $1(1)$ \\
\hline Diversity gain & (1) & (B) 1 & (B) $\mathrm{B} B \mathrm{~B}$ \\
\hline Array gain & (1) (1) & (1) (1) & (B) $13 B$ \\
\hline
\end{tabular}

On the other hand, the challenges to be solved for such configurations (SU-MIMO, MU-MIMO and Massive MIMO) are also discussed and summarized in Table 2.

Table 2. Challenges to be solved for SU-MIMO, MU-MIMO and Massive-MIMO antenna technologies.

\begin{tabular}{|c|c|c|c|}
\hline & \multicolumn{3}{|c|}{ MIMO Antenna Technology } \\
\hline & SU-MIMO & MU-MIMO & Massive MIMO \\
\hline mmWave Bandwidth & (9) & (4) & (P) \\
\hline $\begin{array}{l}\text { Computational } \\
\text { Complexity }\end{array}$ & & & \\
\hline Chanel Estimation & & & \\
\hline Pilot Contamination & & & \\
\hline Signal Detection & & & \\
\hline Precoding & & & \\
\hline $\begin{array}{l}\text { Hardware } \\
\text { Impairment }\end{array}$ & (4) 9 & (4) 9 & (4) (4) \\
\hline User Scheduling & & 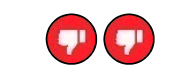 & \\
\hline
\end{tabular}

After discussing the contributions and challenges to be met for the different techniques of MIMO systems, the next section focuses on the technical contributions of some other new methods used in 5G WCNs. 


\section{Technical Contributions of New Methods Used in 5G Wireless Cellular Networks 4.1. High Transmission Speed}

The transmission speed of $5 \mathrm{G}$ is faster than that of $4 \mathrm{G}$ by about 10 to 100 times. Indeed, with values reaching 15 or $20 \mathrm{Gbit} / \mathrm{s}$ and with the use of the cloud, 5G has managed to directly and instantly access programs, files, and remote applications [58-60]. With the increasing use of the cloud, the giant internal memories of devices are no longer essential as information storage, and even software activation have become possible remotely in the cloud [61].

\subsection{Low Latency}

With the arrival of 5G, the latency (defined as the time that elapses from the command on the device until the action is taken) of this technology is greatly reduced compared to that of 4G; indeed, it is of the order of one or two milliseconds (ms) whereas it is of the order of a few tens of $\mathrm{ms}$ for $4 \mathrm{G}(20$ to 40$)[62,63]$. Thanks to this reduced latency, navigation has become more responsive. This acceleration has revolutionized wireless uses in many areas such as audiovisual, machine control, industry, logistics, telemedicine (remote surgical procedures) and even remote and/or automated transport [64].

\subsection{Multiplied Number of Connected Devices}

With 5G, the density of devices that can be connected to the network has increased significantly to reach 1 million per $\mathrm{km}^{2}$. Therefore, the IoT has become more favored because a very large number of devices (100 times more) are instantly connected to the internet and can exchange information [65]. With these developments, 5G has become able to handle real-time data transmission between these devices more quickly [66]. By placing sensors in different points of a city, this principle will lead to smart cities and self-driving cars as well as other services.

\subsection{Network Slicing}

For 5G, one of the most promising options is the implementation of virtual networks or subnetworks, allowing to satisfy, in a programmable way, a connectivity more adapted to specific needs [67]. Here, connections qualified as emergencies are prioritized over others by the application of different latencies, thus leading to the elimination of any overloads. This new feature offered by 5G is called "network slicing" [68-70]. Network slicing enables a dedicated and stable provision of generous bandwidth to meet the requirements of a use case and therefore ensures network security by limiting possible cyber-attacks to such a slice.

\subsection{Energy Efficiency}

As detailed previously, special attention has been paid to revolutionary wireless technologies to meet the ambitious goals of the fifth generation $(5 \mathrm{G})$ cellular networks. The main focus of these efforts has been on challenges in terms of system capacity, data rate, spectral efficiency (SE), average cell throughput and energy efficiency (EE) [71-73]. Indeed, the progress of mobile telephone networks is not limited to the quality of service delivered to customers or to the operational performance, but it is expanding to include the degree to which it takes into account the issues related to energy consumption and even societal and environmental ones. As a result, generation-to-generation progress has managed to achieve an energy efficiency gain of a factor of about 10 [74]. In this context, the developers of 5G networks have focused on integrating energy issues from their design phase in order to reduce the energy consumption of $5 \mathrm{G}$ by a factor of 20 compared to $4 \mathrm{G}$ [75-77]. 


\section{Role of the Massive MIMO and Other Technologies in 5G Wireless Cellular} Networks

\subsection{Massive MIMO}

Massive MIMO systems have attracted attention thanks to their performance in terms of spectral and power efficiency and overall system robustness [5,21,22]. This is mainly due to the possibility of obtaining a capacity of the channels by resorting to a simple correspondence filtering or a receiver so that the inter-channel interference (ICI) is averaged in these systems for the high number of antennas according to the law of large numbers (LLN) [44].

From an economic point of view, the linear requirement of antenna amplifiers is lower in cases where each antenna is affected with less power. Under these conditions, the Massive MIMO system can be designed by combining the components at low cost [54,71].

From an algorithmic and planning standpoint, when operating MU-MIMO in Massive MIMOs, the design of the middle access control (MAC) layer is greatly simplified [55-57].

Although Massive MIMO technology is well judged to be a promising choice for 5G wireless communication networks, several strategic and forward-looking recommendations must be made to meet the main challenges constraining the practical deployment of $5 \mathrm{G}$ and beyond $5 \mathrm{G}(\mathrm{B} 5 \mathrm{G})$.

\section{2. mmWave Technology}

Various works $[32,33]$ have shown that the frequency bands of $5 G$ are not easy to determine precisely and that the $5 \mathrm{G}$ spectrum has been designed to include three main frequency bands which do not necessarily have the same characteristics. Indeed, the lower frequency bands are those responsible for the best mobile coverage, the higher frequency bands are those which provide the best speeds, and the medium bands allow a compromise between the range of the signal and the speed. In this context, we can distinguish the following.

\subsubsection{Low Frequencies}

This range, from around $650 \mathrm{MHz}$ to $1 \mathrm{GHz}$, allows signals to be propagated from a single access point to cover a considerably large area [32]. One of its drawbacks is that if the capacities of the channels are the same for low and high frequencies, the lack of available spectra results in an insufficient number of channels which are capable of delivering the high connection speeds desired to be reached by $5 \mathrm{G}$.

\subsubsection{Mid Frequencies: Sub-6 GHz}

This frequency range is between $1 \mathrm{GHz}$ and $6 \mathrm{GHz}$. The technical justification for this choice lies in the fact that the main gains, in terms of the performance and efficiency of $5 \mathrm{G}$, are based on the portions of the spectrum that are around WiFi [34]. Therefore, service providers can take advantage of frequencies below $6 \mathrm{GHz}$ as a new exploitable spectrum, ensuring wider channels and better latency [29-35]. However, the major problem of this frequency band is that a large part of its spectrum is in free use and that the interference problem caused by the multitude of users must be solved.

\subsubsection{High Frequencies: (Above $6 \mathrm{GHz}$ )}

To further its sophistication and optimization, 5G is increasingly looking to expand its amount of bandwidth to meet its gigabit-scale throughput targets. Unfortunately, the sub-6 $\mathrm{GHz}$ and $1 \mathrm{GHz}$ frequencies are not able to provide enough space. To meet this challenge, a new approach called millimeter waves has been proposed. This technology, whose history began with the studies of J.C. BOSE [78] in 1890, has recently become a reality. Indeed, it was just in 2019 with the rise of $5 \mathrm{G}$ that researchers have renovated this technology in $5 \mathrm{G}$ millimeter wave networks which will obviously be more strategic during the large deployment of $5 \mathrm{G}$. 
Millimeter wave technology is capable of operating between important frequencies, such as 24 and $60 \mathrm{GHz}[32,79]$. Equally, other variable bandwidths that can exceed the $100 \mathrm{GHz}$ limit have been considered as candidate frequencies for the next generation [80]. These considerably high frequency bands allow blazing connection speeds because of the large transmission channels allowed.

- $\quad$ mmWave propagation Model

The received power $\left(\mathrm{P}_{\mathrm{rx}}\right)$ by a wireless system can be expressed as a dependence on the transmitted power $\left(P_{t x}\right)$, the distance between the antennas $(R)$ and the transmit $\left(G_{t x}\right)$ and receive $\left(G_{r x}\right)$ gains according to the Friis Equation (7) [30]:

$$
P_{r x}=P_{t x}+G_{t x}+G_{r x}+20 \log \left(\frac{C}{4 \pi R f}\right)[d B]
$$

Here, $\mathrm{C}$ is the speed of light and $\mathrm{f}$ is the frequency.

The above relationship between the received power and the frequency shows that the higher frequencies generate considerable attenuation compared to the lower ones. Therefore, this technology is more suitable for short distance communications.

- The main constraints of mmWave technology

For mmWave applications, the detrimental effects on the quality of the transmission are materialized by the atmospheric loss in terms of loss of decibels (dB) per kilometer of propagation. These losses mainly depend on humidity, rain, snowfall, air pollution and fog. Recent studies [81], [82] have shown the existence of three different frequency bands depending on the attenuation values. First, the bands between $26 \mathrm{GHz}$ and $38 \mathrm{GHz}$ are deemed the best suited for future $5 \mathrm{G}$ communications because they experience low attenuation. The second bands are those with free-space propagation characteristics and that are comparable to modern cellular frequencies $(73 \mathrm{GHz})$. The third frequency family is unsuitable for indoor short-range communications, such as $60 \mathrm{GHz}$, and is characterized by high attenuation due to rain and absorption of oxygen compared to 28 and $38 \mathrm{GHz}$ frequencies [83].

In addition, the main drawback of the enormous growth of using mmWave for NR application is the decrease in signal power due to molecular absorption. In [79], an in-depth study of the effect of atmospheric conditions on the propagation of mmWave frequencies, supposed to be more used in 5G WCNs, was presented. Particular attention has been paid to the factors of rain, diffraction, air pollutants and foliage and the losses generated on the frequencies centered on 28,30 and $60 \mathrm{GHz}$.

From the above, it can be confirmed that $5 \mathrm{G}$ Sub- 6 bands have satisfactory performance in covering larger geographic areas; however, they are not able to provide a sufficiently fast downlink. In contrast, mmWave 5G technology performs very well in terms of providing top-notch downlink speeds but this comes at the expense of geographic area coverage.

\subsection{Full Duplex}

Previously, communication systems shared resources over time or by frequency division according to the Half-Duplex principle [28]. Under these conditions, the reception and transmission of the signals are ensured in two different frequency bands or in two separate time intervals. Otherwise, the transmission and reception of a signal cannot be done simultaneously at the same frequency. In the case of LTE, the capacity of cellular networks is limited as the communication between the users and the base station is provided by separate uplink and downlink channels which are of equal width [84]. In order to exceed these limits, double the speed compared to half duplex transmissions and increase the spectral efficiency in WCNs, the use of full duplex (FD) technology has been introduced, thanks to the improvement in spectral efficiency that it can bring without any need to widen the frequency band [85]. This is achieved by the fact that, for a specific time slice, the transmission and reception of a signal can be done on the same frequency band. 
Among the advantages of FD technology, a relay is able to exchange data between the destination terminal and the source terminal simultaneously. This significantly improves the delay for FD relay systems. On the security side, another remarkable advantage of FD transmissions is that the coexistence of data transmitted and received on the transmission link at the same time means that the signal, when captured by a foreign third party, is seen as being a mixture of signals that are difficult to decipher [86]. In addition, the ability to transmit and receive on the same spectrum greatly increases or even doubles the flexibility of the spectrum. Another advantage that should not be denied is related to the reduction in latency since the channel status information (CSI) can be received during data transmission.

When it first appeared, FD transmission was deemed impractical in WCSs because of certain technical constraints [85]. On the one hand, the first concern has been related to the very high level of self-interference that can be created when the transceiver sends a signal and receives another together. Otherwise, the processing of two signals (received and transmitted) at the same time makes it very difficult to design a flexible radio transceiver at the same working frequency [84]. To solve these problems and decrease this strong self-interference, the methods proposed to reduce self-interference can be grouped into passive and active cancellations [86].

\subsection{Small Cell}

One of the most promising technologies for 5G WCSs is that of small cells (SC) which can integrate femtocells, picocells and/or microcells [87,88]. This technology has greatly contributed to the management of broadband coverage both outside and inside buildings. Its fundamental principle is to reduce the distance between end-users and access points by integrating access nodes to reduce the coverage radius and the power consumed and improve latency. The use of these different cells has given rise to the appearance of new techniques, such as heterogeneous networks (HetNet) and ultra-dense networks (UDN) [89,90].

Knowing that the main challenges of the $5 \mathrm{G}$ standard are mainly related to certain requirements, such as increasing throughput or spectral capacity, achieving low latency, and reducing energy consumption, etc., the previous technologies have been exploited, individually or combined, to meet $5 \mathrm{G}$ expectations. Such expectations focus on three categories of $5 \mathrm{G}$ uses:

- $\quad$ Enhanced Mobile Broadband (eMBB): This category of use of 5G is designed to meet the growing demand for access to WCNs [91], multimedia services, content and data in terms of coverage and very high speed and bandwidth, especially in urban centers with high user density.

- Massive Machine Type Communications (mMTC): This category [92] is related to the possibility of automating industrial processes with massive machine type communications while ensuring reliability with good connectivity and reduced energy consumption.

- $\quad$ Ultra-Reliable and Low Latency (uRLLC): This scenario $[93,94]$ is essential for applications requiring simultaneously extreme responsiveness and very reliable transmission such as WNCs, remote surgery, autonomous transport, and smart electricity networks [25].

It can be concluded that, among the new technologies on which the 5G standard is based, Massive MIMO systems have succeeded in increasing spectral efficiency and speed. The mmWave technology, in turn, has shown satisfactory efficiency in increasing bandwidth and therefore eliminating the problem of network saturation. In addition, the full duplex, which introduced the use of the bandwidth in a bidirectional way, was also very effective since it doubles the flexibility of the spectrum and reduces the latency to less than 1ms. Regarding small cell technology, it is very efficient in terms of densification and coverage of the network and reduction of interference and consumed energy, especially when combined with mmWave technology. 
The focus of the present work on these four technologies was prompted by the special attention paid to them by the scientific community and the industrialists by considering them as ideal candidates to meet the requirements of $5 \mathrm{G}$.

\section{Recommendations and Future Research Directions}

The recommendations drawn from this in-depth study may, when taken into consideration, be effective for improvements in 5G and even for B5G and 6G. Such recommendations can be summarized as follows:

- To take maximum advantage of the capacity gain of Massive MIMO systems while minimizing the overhead and overhead of CSI acquisition, the use of Time Division Duplex (TDD) and Frequency Division Duplex (FDD) systems is proposed as a solution to be further developed.

- More attention needs to be given to the design of the precoders and detectors for Massive MIMO systems with a number of transmitting antennas equal to or less than that of receiving antennas.

- Furthermore, the search for a compromise between the economy of space and the insertion of a high number of antennas by the theory of matrixes of cubes and the guarantee of the contribution to the gain in channel capacity of all antennas and not just those on the surface of a cubic array.

- One of the most important points is to take into account the phenomena generated by the increase in the antennas of transceivers, such as non-stationary effects and near field effects.

- $\quad$ mmWave Massive MIMO systems are also one of the promising alternatives that need to be further developed to meet the aspirations of current and future cellular communication systems.

- Even with the satisfactory deployment rate and the success of 5G, innovative manipulators in this sector are requested to solve today's challenges by further developing the recent solutions already in use and creating new solutions, such as mobile broadband and scalable IoT.

- Knowing that the natural resonance frequencies of molecules in the mmWave band lead to the degradation of their performance, more efforts should be made concerning the proposal of models, taking into account the effect of atmospheric pollutants according to the air quality index (AQI), especially with the acute air pollution in many cities.

- $\quad$ To ensure outdoor and indoor coverage and overcoming buildings obstacles, reducing the distance between the last access points of the network and users by small cell technology is a solution to be further developed.

- With the spectacular emergence and with the almost infinite potential of artificial intelligence (AI) in various common fields of application, more investment in AI and its giant capacity for learning and reasoning for performance development of intelligent $5 \mathrm{G}$ networks, in terms of efficiency and precision of calculations thus making it possible to manage massive amounts of data, is an inevitable solution. In a related context, it is essential to design robust, flexible and scalable learning frameworks that allow to communicate effectively with high dynamic networks requiring continuous updates.

- The development of hardware components by seeking to reduce their energy consumption and their cost and increase their storage and computing energy is also strongly recommended.

- The concern to adopt techniques of energy management and harvesting as well as wireless energy transfer (to sensors for example) becomes a real challenge for future WCNs, especially with the expansion of air, submarine and spatial infrastructures of these networks.

- More vigilance about the issue of management and security of users' personal data is increasingly a primary challenge. 
- The broadcast nature of mmWave and MIMO signals that will be more used in 5G and B5G gives the possibility to open wireless communication accessibility to both legitimate users and malicious eavesdroppers, jammers and even attackers. To avoid passive interception of transmitted information, the design of effective security mechanisms has to be developed to avoid and combat the attacks.

\section{Conclusions}

To support the increase in transmission rate and the densification of networks, in terms of number of users, especially with the arrival of connected objects, the 5G network revolution has been ensured, in large part, by the integration of mmWave technology and Massive MIMO systems using a very large number of antennas for transmission and/or reception. In addition to these technologies, this paper studied, in depth, the technical concepts and contributions of various recent technologies, such as the full duplex and the small cells, to ensure the empowerment of 5G cellular networks. In addition, although it will take more time for companies interested in this field to fully understand the obstacles and opportunities that such technologies applied to 5G WCNs will bring, this article describes the main trends and challenges. In addition, this paper focuses on the proposal of some recommendations that will serve as motivation for researchers and industrialists interested in 5G and future technologies to take into account the consideration of the future generations of WCNs as a balanced ecosystem synchronizing technical capacities with socio-economic, environmental and other concerns. The findings of this paper are a valuable guidance for academia, researchers and industrialists in new and future WCNs fields.

Funding: The author would like to thank the deanship of scientific research at University of Ha'il for funding this work under the Research Project (BA-2007).

Institutional Review Board Statement: Not applicable.

Informed Consent Statement: Not applicable.

Data Availability Statement: The data presented in this study are available within the article.

Acknowledgments: The author acknowledges the financial support of the University of Ha'il (UOH) for the support of this work through the Research Project \# BA-2007.

Conflicts of Interest: The author declares no conflict of interest.

\section{Nomenclature and Abbreviations}

$\begin{array}{ll}\text { 1G } & \text { Fifth-generation } \\ 2 \mathrm{G} & \text { Second-generation } \\ 3 \mathrm{G} & \text { Third-generation } \\ 4 \mathrm{G} & \text { Fourth-generation } \\ 5 \mathrm{G} & \text { Fifth-generation } \\ 6 \mathrm{G} & \text { Sixth-generation } \\ \text { MIMO } & \text { Multiple Input, Multiple Output } \\ \text { WCS } & \text { Wireless communication system } \\ \text { WCNs } & \text { Wireless cellular network } \\ \text { QoS } & \text { Quality of service } \\ \text { M2M } & \text { Machine-to-machine } \\ \text { ITU } & \text { International Telecommunication Union } \\ \text { WLAN } & \text { Wireless local area networks } \\ \text { DODs } & \text { Direction of departures } \\ \text { DOAs } & \text { Direction of arrivals } \\ \text { OFDM } & \text { Orthogonal frequency division multiplexing } \\ \text { BS } & \text { Base station } \\ \text { ZF } & \text { Zero-forcing } \\ \text { IoT } & \text { Internet of things }\end{array}$




$\begin{array}{ll}\text { NR } & \text { New radio } \\ \text { gNB } & \text { Next generation Node B } \\ \text { UE } & \text { User equipment } \\ \text { URLLCs } & \text { Ultra-reliable and low-latency communications } \\ \text { PACE } & \text { Pilot-assisted channel estimation } \\ \text { SISO } & \text { Single Input, Single Output } \\ \text { SIMO } & \text { Single Input, Multiple Output } \\ \text { MISO } & \text { Multiple Input, Single Output } \\ \text { CSI } & \text { Channel state information } \\ \text { SNR } & \text { Signal to noise ratio } \\ \text { SDM } & \text { Spatial Division Multiplexing } \\ \text { STBC } & \text { Space-Time Block Code } \\ \text { LTE } & \text { Long term evolution } \\ \text { LTEA } & \text { Advanced LTE } \\ \text { SU-MIMO } & \text { Single-user MIMO } \\ \text { MU-MIMO } & \text { Multi-user MIMO } \\ \text { SE } & \text { Spectral efficiency } \\ \text { EE } & \text { Energy efficiency } \\ \text { LLN } & \text { Law of large numbers } \\ \text { MAC } & \text { Middle access control } \\ \text { B5G } & \text { Beyond 5G } \\ \text { TDD } & \text { Time Division Duplex } \\ \text { FDD } & \text { Frequency Division Duplex } \\ \text { FD } & \text { Full-Duplex } \\ \text { HD } & \text { Half-Duplex } \\ \text { mmWave } & \text { Millimeter wave } \\ \text { AI } & \text { Artificial Intelligence } \\ \text { AQI } & \text { Air Quality Index } \\ \text { eMBB } & \text { Enhanced Mobile Broadband } \\ \text { mMTC } & \text { Massive Machine Type Communications } \\ \text { SC } & \text { Small Cell } \\ \text { HetNet } & \text { Heterogeneous Networks } \\ \text { UDN } & \text { Ultra Dense Networks } \\ \text { PA } & \text { Power Amplifier } \\ \text { ICI } & \text { Inter-channel interference } \\ & \end{array}$

\section{References}

1. International Telecommunication Union. IMT Traffic Estimates for the Years 2020 to 2030. 2015. Available online: https: / / www.itu.int/dms_pub/itu-r/opb/rep/R-REP-M.2370-2015-PDF-E.pdf (accessed on 10 March 2021).

2. Khan, M.S.; Iftikhar, A.; Shubair, R.M.; Capobianco, A.-D.; Braaten, B.D.; Anagnostou, D.E. Eight-Element Compact UWBMIMO/Diversity Antenna with WLAN Band Rejection for 3G/4G/5G Communications. IEEE Open J. Antennas Propag. 2020, 1, 196-206. [CrossRef]

3. Sharma, P. Evolution of mobile wireless communication networks: $1 \mathrm{G}$ to $5 \mathrm{G}$ as well as future prospective of next generation communication network. Int. J. Comput. Sci. Mobile Comput. 2013, 2, 47-53.

4. Han, J.; Kwon, K. I/Q Balance-Enhanced Wideband Receiver Front-End for 2G/3G/4G/5G NR Cellular Applications. IEEE Trans. Circuits Syst. I Regul. Pap. 2020, 67, 1881-1891. [CrossRef]

5. Liu, Y.; Wang, C.-X.; Huang, J.; Sun, J.; Zhang, W. Novel 3-D Nonstationary MmWave Massive MIMO Channel Models for 5G High-Speed Train Wireless Communications. IEEE Trans. Veh. Technol. 2019, 68, 2077-2086. [CrossRef]

6. Khalid, N.; Akan, O.B. Experimental Throughput Analysis of Low-THz MIMO Communication Channel in 5G Wireless Networks. IEEE Wirel. Commun. Lett. 2016, 5, 616-619. [CrossRef]

7. Li, X.; Qin, N.; Sun, T. Interference coordination for FD-MIMO cellular network with D2D communications underlaying. China Commun. 2018, 15, 75-88.

8. Patwary, M.N.; Nawaz, S.J.; Rahman, A.; Sharma, S.K.; Rashid, M.; Barnes, S.J. The Potential Short- and Long-Term Disruptions and Transformative Impacts of 5G and Beyond Wireless Networks: Lessons Learnt from the Development of a 5G Testbed Environment. IEEE Access 2020, 8, 11352-11379. [CrossRef]

9. Meng, M.; Xiao, W.; He, T.; Tao, Y.; Tan, K.; Zhang, J.; Wang, W. BeamRaster: A Practical Fast Massive MU-MIMO System with Pre-Computed Precoders. IEEE Trans. Mob. Comput. 2019, 18, 1014-1027. [CrossRef]

10. IEEE Standards Association. IEEE 1711.2-2019_IEEE Standard for Secure SCADA Communications Protocol (SSCP); IEEE Standards Association: Piscataway, NJ, USA, 2020; pp. 1-37. 
11. Melo, P.; Pimentel, C.; Alajaji, F. LDPC Decoding Over Non-Binary Queue-Based Burst Noise Channels. IEEE Trans. Veh. Technol. 2016, 65, 452-457. [CrossRef]

12. Mathur, R.; Dwari, S. Compact planar reconfigurable UWB-MIMO antenna with on-demand worldwide interoperability for microwave access/wireless local area network rejection. IET Microw. Antennas Propag. 2019, 13, 1684-1689. [CrossRef]

13. Fan, P.; Cui, X.; Lu, M. Space and frequency diversity characterization of mobile GNSS receivers in multipath fading channels. Tsinghua Sci. Technol. 2020, 25, 294-301. [CrossRef]

14. Xu, C.; Sugiura, S.; Ng, S.X.; Zhang, P.; Wang, L.; Hanzo, L. Two Decades of MIMO Design Tradeoffs and Reduced-Complexity MIMO Detection in Near-Capacity Systems. IEEE Access 2017, 5, 18564-18632. [CrossRef]

15. He, Q.; Wang, Z.; Hu, J.; Blum, R.S. Performance Gains from Cooperative MIMO Radar and MIMO Communication Systems. IEEE Signal Process. Lett. 2019, 26, 194-198. [CrossRef]

16. Qian, J.; Lops, M.; Zheng, L.; Wang, X.; He, Z. Joint System Design for Coexistence of MIMO Radar and MIMO Communication. IEEE Trans. Signal Process. 2018, 66, 3504-3519. [CrossRef]

17. Cai, X.; Wang, X.; Huang, Z.; Wang, F. Single-Channel Blind Source Separation of Communication Signals Using Pseudo-MIMO Observations. IEEE Commun. Lett. 2018, 22, 1616-1619. [CrossRef]

18. Liu, F.; Masouros, C.; Li, A.; Sun, H.; Hanzo, L. MU-MIMO Communications with MIMO Radar: From Co-Existence to Joint Transmission. IEEE Trans. Wirel. Commun. 2018, 17, 2755-2770. [CrossRef]

19. Missaoui, N.; Kammoun, I.; Siala, M. Efficient User Identification and Semi-Blind Channel Estimation for MU-MIMO Systems. IEEE Wirel. Commun. Lett. 2017, 6, 150-153. [CrossRef]

20. Moazzen, H.; Mohammadi, A.; Majidi, M. Performance Analysis of Linear Precoded MU-MIMO-OFDM Systems with Nonlinear Power Amplifiers and Correlated Channel. IEEE Trans. Commun. 2019, 67, 6753-6765. [CrossRef]

21. Bjornson, E.; Sanguinetti, L.; Hoydis, J.; Debbah, M. Optimal Design of Energy-Efficient Multi-User MIMO Systems: Is Massive MIMO the Answer? IEEE Trans. Wirel. Commun. 2015, 14, 3059-3075. [CrossRef]

22. Du, J.; Han, M.; Jin, L.; Hua, Y.; Li, X. Semi-Blind Receivers for Multi-User Massive MIMO Relay Systems Based on Block Tucker2-PARAFAC Tensor Model. IEEE Access 2020, 8, 32170-32186. [CrossRef]

23. Lee, W. Resource Allocation for Multi-Channel Underlay Cognitive Radio Network Based on Deep Neural Network. IEEE Commun. Lett. 2018, 22, 1942-1945. [CrossRef]

24. Jacobsson, S.; Durisi, G.; Coldrey, M.; Studer, C. Linear Precoding With Low-Resolution DACs for Massive MU-MIMO-OFDM Downlink. IEEE Trans. Wirel. Commun. 2019, 18, 1595-1609. [CrossRef]

25. Zeng, J.; Lv, T.; Liu, R.P.; Su, X.; Guo, Y.J.; Beaulieu, N.C. Enabling Ultrareliable and Low-Latency Communications Under Shadow Fading by Massive MU-MIMO. IEEE Internet Things J. 2020, 7, 234-246. [CrossRef]

26. Zeng, J.; Lv, T.; Liu, R.P.; Su, X.; Beaulieu, N.C.; Guo, Y.J. Linear Minimum Error Probability Detection for Massive MU-MIMO With Imperfect CSI in URLLC. IEEE Trans. Veh. Technol. 2019, 68, 11384-11388. [CrossRef]

27. Chu, L.; Wen, F.; Qiu, R.C. Eigen-Inference Precoding for Coarsely Quantized Massive MU-MIMO System with Imperfect CSI. IEEE Trans. Veh. Technol. 2019, 68, 8729-8743. [CrossRef]

28. Vogt, H.; Awan, Z.H.; Sezgin, A. Secret-Key Generation: Full-Duplex Versus Half-Duplex Probing. IEEE Trans. Commun. 2019, 67, 639-652. [CrossRef]

29. Chen, H.-D.; Tsai, Y.-C.; Sim, C.; Kuo, C. Broadband Eight-Antenna Array Design for Sub-6 GHz 5G NR Bands Metal-Frame Smartphone Applications. IEEE Antennas Wirel. Propag. Lett. 2020, 19, 1078-1082. [CrossRef]

30. Zada, M.; Shah, I.A.; Yoo, H. Integration of Sub-6-GHz and mm-Wave Bands With a Large Frequency Ratio for Future 5G MIMO Applications. IEEE Access 2021, 9, 11241-11251. [CrossRef]

31. Zhang, X.; Wang, J.; Poor, H.V. Heterogeneous Statistical-QoS Driven Resource Allocation Over mmWave Massive-MIMO Based 5G Mobile Wireless Networks in the Non-Asymptotic Regime. IEEE J. Sel. Areas Commun. 2019, 37, 2727-2743. [CrossRef]

32. Taheri, M.M.S.; Abdipour, A.; Zhang, S.; Pedersen, G.F. Integrated Millimeter-Wave Wideband End-Fire 5G Beam Steerable Array and Low-Frequency 4G LTE Antenna in Mobile Terminals. IEEE Trans. Veh. Technol. 2019, 68, 4042-4046. [CrossRef]

33. Ozpinar, H.; Aksimsek, S.; Tokan, N.T. A Novel Compact, Broadband, High Gain Millimeter-Wave Antenna for 5G Beam Steering Applications. IEEE Trans. Veh. Technol. 2020, 69, 2389-2397. [CrossRef]

34. Ciydem, M.; Miran, E.A. Dual-Polarization Wideband Sub-6 GHz Suspended Patch Antenna for 5G Base Station. IEEE Antennas Wirel. Propag. Lett. 2020, 19, 1142-1146. [CrossRef]

35. Serghiou, D.; Khalily, M.; Singh, V.; Araghi, A.; Tafazolli, R. Sub-6 GHz Dual-Band $8 \times 8$ MIMO Antenna for 5G Smartphones. IEEE Antennas Wirel. Propag. Lett. 2020, 19, 1546-1550. [CrossRef]

36. Jin, G.; Deng, C.; Xu, Y.; Yang, J.; Liao, S. Differential Frequency-Reconfigurable Antenna Based on Dipoles for Sub-6 GHz 5G and WLAN Applications. IEEE Antennas Wirel. Propag. Lett. 2020, 19, 472-476. [CrossRef]

37. Ikram, M.; Nguyen-Trong, N.; Abbosh, A.M. Common-Aperture Sub-6 GHz and Millimeter-Wave 5G Antenna System. IEEE Access 2020, 8, 199415-199423. [CrossRef]

38. Damodaran, S.; Kumar, T.K.S.; Sudheer, A.P. Model-Matching Fractional-Order Controller Design Using AGTM/AGMP Matching Technique for SISO/MIMO Linear Systems. IEEE Access 2019, 7, 41715-41728. [CrossRef]

39. Lei, Y.; Xu, K.; Li, J.; Meng, Z.; Wu, R.; Wan, Z.; Fan, Y.; Zhang, W.; Yin, F.; Dai, Y. Feasibility of Space-Division-Multiplexed Transmission of IEEE $802.11 \mathrm{n} /$ ac-Compliant Wireless MIMO Signals Over OM3 Multimode Fiber. J. Light. Technol. 2018, 36, 2076-2082. [CrossRef] 
40. Namitha, A.S.; Sameer, S.M. A bandwidth efficient selective mapping technique for the PAPR reduction in spatial multiplexing MIMO-OFDM wireless communication system. Phys. Commun. 2017, 25, 128-138. [CrossRef]

41. Poudel, B.; Oshima, J.; Kobayashi, H.; Iwashita, K. MIMO detection using a deep learning neural network in a mode division multiplexing optical transmission system. Opt. Commun. 2019, 440, 41-48. [CrossRef]

42. Winzer, P.J. Optical MIMO-SDM: From interface rates and fiber capacity scaling to physical-layer security. In Proceedings of the 2015 17th International Conference on Transparent Optical Networks (ICTON), Budapest, Hungary, 5-9 July 2015; p. 1. [CrossRef]

43. Han, J.; Zhang, J.; Zhao, Y.; Gu, W. Channel capacity and space-time block coding for coherent optical MIMO multi-mode fiber links. Optik 2013, 124, 922-927. [CrossRef]

44. Busari, S.A.; Huq, K.M.S.; Mumtaz, S.; Dai, L.; Rodriguez, J. Millimeter-Wave Massive MIMO Communication for Future Wireless Systems: A Survey. IEEE Commun. Surv. Tutor. 2018, 20, 836-869. [CrossRef]

45. IEEE Standards Association. IEEE Standard for Air Interface for Broadband Wireless Access Systems-Amendment 4: Fixed and Mobile Wireless Access in Channel Bandwidth up to 1.25 MHz; IEEE Std 802.16s-2017 (Amendment to IEEE Std 802.16-2012 as amended by IEEE Std 802.16p-2012, IEEE Std 802.16n-2013, IEEE Std 802.16q-2015); IEEE Standards Association: Piscataway, NJ, USA, 2017; pp. $1-42$.

46. Karmakar, R.; Chattopadhyay, S.; Chakraborty, S. Impact of IEEE 802.11n/ac PHY/MAC High Throughput Enhancements on Transport and Application Protocols-A Survey. IEEE Commun. Surv. Tutor. 2017, 19, 2050-2091. [CrossRef]

47. Ko, H.; Lee, G.; Suh, D.; Pack, S.; Shen, X. An Optimized and Distributed Data Packet Forwarding Scheme in LTE/LTE-A Networks. IEEE Trans. Veh. Technol. 2016, 65, 3462-3473. [CrossRef]

48. Vehkapera, M.; Riihonen, T.; Girnyk, M.A.; Bjornson, E.; Debbah, M.; Rasmussen, L.K.; Wichman, R. Asymptotic Analysis of SU-MIMO Channels with Transmitter Noise and Mismatched Joint Decoding. IEEE Trans. Commun. 2015, 63, 749-765. [CrossRef]

49. Xue, Q.; Fang, X.; Wang, C.-X. Beamspace SU-MIMO for Future Millimeter Wave Wireless Communications. IEEE J. Sel. Areas Commun. 2017, 35, 1564-1575. [CrossRef]

50. Muthalagu, R. Non-Linear and Non-Iterative Based Transceiver Design for SU-MIMO Systems. J. Commun. Inf. Netw. 2018, 3, 84-92. [CrossRef]

51. Kosek-Szott, K.; Cuka, G. Consequences of Performing DL MU-MIMO Transmissions with TXOP Sharing for QoS Provisioning in IEEE 802.11ac Networks. IEEE Commun. Lett. 2018, 22, 606-609. [CrossRef]

52. Ahn, Y.; Kim, T.; Lee, C. A Beam Steering Based Hybrid Precoding for MU-MIMO mmWave Systems. IEEE Commun. Lett. 2017, 21, 2726-2729. [CrossRef]

53. Ma, Y.; Yamani, A.; Yi, N.; Tafazolli, R. Low-Complexity MU-MIMO Nonlinear Precoding Using Degree-2 Sparse Vector Perturbation. IEEE J. Sel. Areas Commun. 2016, 34, 497-509. [CrossRef]

54. Abdallah, A.; Mansour, M.M. Efficient Angle-Domain Processing for FDD-Based Cell-Free Massive MIMO Systems. IEEE Trans. Commun. 2020, 68, 2188-2203. [CrossRef]

55. You, L.; Chen, X.; Song, X.; Jiang, F.; Wang, W.; Gao, X.; Fettweis, G. Network Massive MIMO Transmission Over Millimeter-Wave and Terahertz Bands: Mobility Enhancement and Blockage Mitigation. IEEE J. Sel. Areas Commun. 2020, 38, 2946-2960. [CrossRef]

56. Liu, P.; Li, Y.; Zhang, Z. Multiple Fan-Beam Antenna Array for Massive MIMO Applications. J. Commun. Inf. Netw. 2018, 3, 38-42. [CrossRef]

57. Albreem, M.A.; Juntti, M.; Shahabuddin, S. Massive MIMO Detection Techniques: A Survey. IEEE Commun. Surv. Tutor. 2019, 21, 3109-3132. [CrossRef]

58. Wang, Y.; Wang, Y.; Zhang, S.; Cen, H. Channel Tracking and Transmission Design in 5G Large-Scale MIMO System. IEEE Access 2019, 7, 62032-62041. [CrossRef]

59. Kim, K.O.; Doo, K.-H.; Lee, H.H.; Kim, S.H.; Park, H.; Oh, J.-Y.; Chung, H.S.; Doo, K.H. High Speed and Low Latency Passive Optical Network for 5G Wireless Systems. J. Light. Technol. 2019, 37, 2873-2882. [CrossRef]

60. Sung, M.; Kim, J.; Kim, E.-S.; Cho, S.-H.; Won, Y.-J.; Lim, B.-C.; Pyun, S.-Y.; Lee, H.; Lee, J.K.; Lee, J.H. RoF-Based Radio Access Network for 5G Mobile Communication Systems in 28 GHz Millimeter-Wave. J. Lightwave Technol. 2020, 38, 409-420. [CrossRef]

61. Sezgin, I.C.; Dahlgren, M.; Eriksson, T.; Coldrey, M.; Larsson, C.; Gustavsson, J.; Fager, C. A Low-Complexity Distributed-MIMO Testbed Based on High-Speed Sigma-Delta-Over-Fiber. IEEE Trans. Microw. Theory Tech. 2019, 67, 2861-2872. [CrossRef]

62. Mutalemwa, L.C.; Shin, S. A Classification of the Enabling Techniques for Low Latency and Reliable Communications in 5G and Beyond: AI-Enabled Edge Caching. IEEE Access 2020, 8, 205502-205533. [CrossRef]

63. Parvez, I.; Rahmati, A.; Guvenc, I.; Sarwat, A.I.; Dai, H. A Survey on Low Latency Towards 5G: RAN, Core Network and Caching Solutions. IEEE Commun. Surv. Tutor. 2018, 20, 3098-3130. [CrossRef]

64. Rico, D.; Merino, P. A Survey of End-to-End Solutions for Reliable Low-Latency Communications in 5G Networks. IEEE Access 2020, 8, 192808-192834. [CrossRef]

65. $\mathrm{Xu}, \mathrm{T}$.; Darwazeh, I. Non-Orthogonal Narrowband Internet of Things: A Design for Saving Bandwidth and Doubling the Number of Connected Devices. IEEE Internet Things J. 2018, 5, 2120-2129. [CrossRef]

66. Huang, J.; Qian, Y.; Hu, R.Q. Secure and Efficient Privacy-Preserving Authentication Scheme for 5G Software Defined Vehicular Networks. IEEE Trans. Veh. Technol. 2020, 69, 8542-8554. [CrossRef]

67. Mei, C.; Liu, J.; Li, J.; Zhang, L.; Shao, M. 5G network slices embedding with sharable virtual network functions. J. Commun. Networks 2020, 22, 415-427. [CrossRef] 
68. Song, C.; Zhang, M.; Zhan, Y.; Wang, D.; Guan, L.; Liu, W.; Zhang, L.; Xu, S. Hierarchical Edge Cloud Enabling Network Slicing for 5G Optical Fronthaul. J. Opt. Commun. Netw. 2019, 11, B60-B70. [CrossRef]

69. Sattar, D.; Matrawy, A. Optimal Slice Allocation in 5G Core Networks. IEEE Netw. Lett. 2019, 1, 48-51. [CrossRef]

70. Li, X.; Guo, C.; Gupta, L.; Jain, R. Efficient and Secure 5G Core Network Slice Provisioning Based on VIKOR Approach. IEEE Access 2019, 7, 150517-150529. [CrossRef]

71. Patcharamaneepakorn, P.; Wu, S.; Wang, C.-X.; Aggoune, E.-H.M.; Alwakeel, M.M.; Ge, X.; Di Renzo, M. Spectral, Energy, and Economic Efficiency of 5G Multicell Massive MIMO Systems with Generalized Spatial Modulation. IEEE Trans. Veh. Technol. 2016, 65, 9715-9731. [CrossRef]

72. Biyabani, S.R.; Khan, R.; Alam, M.M.; Biyabani, A.A.; McCune, E. Energy Efficiency Evaluation of Linear Transmitters for 5G NR Wireless Waveforms. IEEE Trans. Green Commun. Netw. 2019, 3, 446-454. [CrossRef]

73. Cheng, W.; Zhang, X.; Zhang, H. Statistical-QoS Driven Energy-Efficiency Optimization Over Green 5G Mobile Wireless Networks. IEEE J. Sel. Areas Commun. 2016, 34, 3092-3107. [CrossRef]

74. Hello Future. La 5G: L’Efficacité Énergétique “By Design”. 2020. Available online: https://hellofuture.orange.com/fr/la-5glefficacite-energetique-by-design/ (accessed on 12 March 2021).

75. Patcharamaneepakorn, P.; Wang, C.-X.; Fu, Y.; Aggoune, E.-H.M.; Alwakeel, M.M.; Tao, X.; Ge, X. Quadrature Space-Frequency Index Modulation for Energy-Efficient 5G Wireless Communication Systems. IEEE Trans. Commun. 2018, 66, 3050-3064. [CrossRef]

76. Chang, K.-C.; Chu, K.-C.; Wang, H.-C.; Lin, Y.-C.; Pan, J.-S. Energy Saving Technology of 5G Base Station Based on Internet of Things Collaborative Control. IEEE Access 2020, 8, 32935-32946. [CrossRef]

77. Hellaoui, H.; Koudil, M.; Bouabdallah, A. Energy Efficiency in Security of 5G-Based IoT: An End-to-End Adaptive Approach. IEEE Internet Things J. 2020, 7, 6589-6602. [CrossRef]

78. Emerson, D. The work of Jagadis Chandra Bose: 100 years of millimeter-wave research. IEEE Trans. Microw. Theory Tech. 1997, 45, 2267-2273. [CrossRef]

79. Banday, Y.; Rather, G.M.; Begh, G.R. Effect of atmospheric absorption on millimetre wave frequencies for 5G cellular networks. IET Commun. 2019, 13, 265-270. [CrossRef]

80. Lota, J.; Sun, S.; Rappaport, T.S.; Demosthenous, A. 5G Uniform Linear Arrays with Beamforming and Spatial Multiplexing at 28, 37, 64, and 71 GHz for Outdoor Urban Communication: A Two-Level Approach. IEEE Trans. Veh. Technol. 2017, 66, 9972-9985. [CrossRef]

81. Lou, H.; Ge, X.; Li, Q. The New Purity and Capacity Models for the OAM-mmWave Communication Systems Under Atmospheric Turbulence. IEEE Access 2019, 7, 129988-129996. [CrossRef]

82. Rappaport, T.S.; Sun, S.; Mayzus, R.; Zhao, H.; Azar, Y.; Wang, K.; Wong, G.N.; Schulz, J.K.; Samimi, M.; Gutierrez, F. Millimeter Wave Mobile Communications for 5G Cellular: It Will Work! IEEE Access 2013, 1, 335-349. [CrossRef]

83. Liu, P.; Di Renzo, M.; Springer, A. Line-of-Sight Spatial Modulation for Indoor mmWave Communication at 60 GHz. IEEE Trans. Wirel. Commun. 2016, 15, 7373-7389. [CrossRef]

84. Fakih, M.A.; Diallo, A.; Le Thuc, P.; Staraj, R.; Mourad, O.; Rachid, E.A. Optimization of Efficient Dual Band PIFA System for MIMO Half-Duplex 4G/LTE and Full-Duplex 5G Communications. IEEE Access 2019, 7, 128881-128895. [CrossRef]

85. Afifi, W.; Abdel-Rahman, M.J.; Krunz, M.; MacKenzie, A.B. Full-Duplex or Half-Duplex: A Bayesian Game for Wireless Networks with Heterogeneous Self-Interference Cancellation Capabilities. IEEE Trans. Mob. Comput. 2018, 17, 1076-1089. [CrossRef]

86. Wang, D.; Wang, M.; Zhu, P.; Li, J.; Wang, J.; You, X. Performance of Network-Assisted Full-Duplex for Cell-Free Massive MIMO. IEEE Trans. Commun. 2020, 68, 1464-1478. [CrossRef]

87. Zaidi, S.M.A.; Manalastas, M.; Farooq, H.; Imran, A. Mobility Management in Emerging Ultra-Dense Cellular Networks: A Survey, Outlook, and Future Research Directions. IEEE Access 2020, 8, 183505-183533. [CrossRef]

88. Mowla, M.; Ahmad, I.; Habibi, D.; Phung, Q.V.; Phung, V. Energy Efficient Backhauling for 5G Small Cell Networks. IEEE Trans. Sustain. Comput. 2019, 4, 279-292. [CrossRef]

89. Zhu, A.; Ma, M.; Guo, S.; Yu, S.; Yi, L. Adaptive Multi-Access Algorithm for Multi-Service Edge Users in 5G Ultra-Dense Heterogeneous Networks. IEEE Trans. Veh. Technol. 2021. [CrossRef]

90. Jo, Y.; Kim, H.; Lim, J.; Hong, D. Self-Optimization of Coverage and System Throughput in 5G Heterogeneous Ultra-Dense Networks. IEEE Wirel. Commun. Lett. 2020, 9, 285-288. [CrossRef]

91. Liu, G.; Hou, X.; Jin, J.; Wang, F.; Wang, Q.; Hao, Y.; Huang, Y.; Wang, X.; Xiao, X.; Deng, A. 3-D-MIMO With Massive Antennas Paves the Way to 5G Enhanced Mobile Broadband: From System Design to Field Trials. IEEE J. Sel. Areas Commun. 2017, 35, 1222-1233. [CrossRef]

92. Sim, Y.; Cho, D.-H. Performance Evaluation of Partially Clustered Access Scheme for Massive Machine Type Communications. IEEE Commun. Lett. 2020, 24, 626-629. [CrossRef]

93. Popovski, P.; Stefanovic, C.; Nielsen, J.J.; De Carvalho, E.; Angjelichinoski, M.; Trillingsgaard, K.F.; Bana, A.-S. Wireless Access in Ultra-Reliable Low-Latency Communication (URLLC). IEEE Trans. Commun. 2019, 67, 5783-5801. [CrossRef]

94. Vu, T.K.; Liu, C.-F.; Bennis, M.; Debbah, M.; Latva-Aho, M.; Hong, C.S. Ultra-Reliable and Low Latency Communication in mmWave-Enabled Massive MIMO Networks. IEEE Commun. Lett. 2017, 21, 2041-2044. [CrossRef] 